YITP-SB-07-17

\title{
Path Integration in QCD with Arbitrary Space-Dependent Static Color Potential
}

\author{
Gouranga C. Nayak ${ }^{1, *}$ \\ 1 C. N. Yang Institute for Theoretical Physics, \\ Stony Brook University, SUNY, Stony Brook, NY 11794-3840, USA
}

(Dated: October 26, 2018)

\begin{abstract}
We perform the path integral for a quark (antiquark) in the presence of an external background chromo-electric $\mathrm{SU}(3)$ gauge field $E^{a}\left(x^{1}=x\right)$ with arbitrary color index $a=1,2, \ldots 8$ and obtain an exact non-perturbative expression for the generating functional. The only nonzero field strength component considered is $E^{a}=F_{01}^{a}$ which is allowed to depend on a single spatial coordinate $x^{1}=x$. We show that such a path integration is possible even if one can not solve the Dirac equation in the presence of arbitrary space-dependent potential. This result crucially depends on the validity of the shift conjecture which has not yet been established. It may be possible to further explore this path integral technique to study non-perturbative bound state formation.

PACS numbers: PACS: 12.38.-t, 11.15.-q, 11.15.Tk, 11.15.Me
\end{abstract}

*Electronic address: nayak@max2.physics.sunysb.edu

Typeset by REVTEX 


\section{INTRODUCTION}

In QED and in Newtonian gravity the potential (due to charge and mass respectively) is inversely proportional to distance. However, the exact form of the potential due to color charge is not known in QCD. This is a fundamental problem in physics. Quarks and gluons which carry color charges are confined inside hadrons.

Lattice QCD simulation attempts to compute the form of the static potential between quark and antiquark inside quarkonium [1, 2]. The static potential inside heavy quarkonia can be approximated to be a Coulomb plus linear form [3]. However, the exact form of the potential inside light $q \bar{q}$ mesons and inside other hadrons is expected to be quite complicated. The long distance confinement physics is non-perturbative, and one can not perform path integrations analytically. On the other hand the lattice QCD method implements extensive numerical simulations and hence the physical picture is not as clear as it could be if one had explicit analytic results. From this point of view path integration in the presence of arbitrary space-dependent static color potential would be desirable.

In this paper we present an analytical method to perform the path integral for a quark (antiquark) in the presence of arbitrary space-dependent static color potential $A_{0}^{a}(x)(=$ $\left.-\int d x E^{a}(x)\right)$ with arbitrary color index $a=1,2, . .8$ in $\mathrm{SU}(3)$. We show that such a path integration is possible even if one can not solve the Dirac equation in the presence of arbitrary space-dependent potential. This result crucially depends on the validity of the shift conjecture which has not yet been established. Unlike QED, the above potential in QCD is motivated because the color quark-antiquark potential may be one dimensional. In particular we obtain the following exact non-perturbative expression for the generating functional for a quark (antiquark) in the presence of arbitrary space-dependent static color potential $A_{0}^{a}(x)=-\int d x E^{a}(x):$

$$
\begin{aligned}
& \frac{Z[A]}{Z[0]}=\frac{\int[d \bar{\psi}][d \psi] e^{i \int d^{4} x \bar{\psi}^{j}(x)\left(\delta_{j k} \not{p}-g T_{j k}^{a} \not^{a}-\delta_{j k} m\right) \psi^{k}(x)}}{\int[d \bar{\psi}][d \psi] e^{i \int d^{4} x \bar{\psi}^{j}(x)(\not{p}-m) \psi^{k}(x)}}= \\
& \exp \left\{\frac{i}{8 \pi^{2}} \sum_{j=1}^{3} \int d t \int d^{3} x \int_{0}^{\infty} \frac{d s}{s^{2}} e^{-i s\left(m^{2}-i \epsilon\right)}\left[g \Lambda_{j}(x) \operatorname{coth}\left(g s \Lambda_{j}(x)\right)-\frac{1}{3} s g^{2} \Lambda_{j}^{2}(x)-\frac{1}{s}\right]\right\} .
\end{aligned}
$$

The gauge invariant $\Lambda_{j}(x)$ 's are given by

$$
\Lambda_{1}(x)=\sqrt{\frac{C_{1}(x)}{3}} \cos \theta(x), \quad \Lambda_{2,3}(x)=\sqrt{\frac{C_{1}(x)}{3}} \cos \left(\frac{2 \pi}{3} \pm \theta(x)\right), \quad \cos ^{2} 3 \theta(x)=\frac{3 C_{2}(x)}{C_{1}^{3}(x)}
$$


where $C_{1}(x)=\left[E^{a}(x) E^{a}(x)\right]$ and $C_{2}(x)=\left[d_{a b c} E^{a}(x) E^{b}(x) E^{c}(x)\right]^{2}$ are two independent space-dependent casimir/gauge invariants in $\mathrm{SU}(3)$. The color indices $a, b, c=1,2, \ldots 8$.

We will present a derivation of eq. (1) in this paper. It may be possible to further explore this path integral technique to study non-perturbative bound state formation, for example, by studying various non-perturbative correlation functions.

\section{PATH INTEGRATION WITH ARBITRARY SPACE-DEPENDENT STATIC COLOR POTENTIAL}

The Lagrangian density for quark in a classical chromofield $A_{\mu}^{a}$ is given by

$$
\mathcal{L}=\bar{\psi}^{j}\left[\left(\delta_{j k} \hat{p}-g T_{j k}^{a} \mathcal{A}^{a}\right)-m \delta_{j k}\right] \psi^{k}=\bar{\psi}^{j} M_{j k}[A] \psi^{k}
$$

where $\hat{p}_{\mu}=\frac{1}{i} \frac{\partial}{\partial x^{\mu}}$ is the momentum operator and $T_{j k}^{a}$ is the generator in the fundamental representation of gauge group $\mathrm{SU}(3)$ with $a=1,2 \ldots 8$ and $j, k=1,2,3$. The generating functional is given by

$$
\frac{Z[A]}{Z[0]}=\frac{\int[d \bar{\psi}][d \psi] e^{i \int d^{4} x \bar{\psi}^{j} M_{j k}[A] \psi^{k}}}{\int[d \bar{\psi}][d \psi] e^{i \int d^{4} x \bar{\psi}^{j} M_{j k}[0] \psi^{k}}}=\frac{\operatorname{Det}[\mathrm{M}[\mathrm{A}]]}{\operatorname{Det}[\mathrm{M}[0]]}=e^{i W}
$$

This gives

$$
W=-\frac{i}{2} \operatorname{Tr} \ln \left[\left(\delta_{j k} \hat{p}-g T_{j k}^{a} \not^{a}\right)^{2}-m^{2} \delta_{j k}\right]+\frac{i}{2} \operatorname{Tr} \ln \left[\delta_{j k}\left(\hat{p}^{2}-m^{2}\right)\right]
$$

where

$$
\operatorname{Tr} \mathcal{O}=\operatorname{tr}_{\text {Dirac }} \operatorname{tr}_{\text {color }} \int d t \int d x \int d y \int d z<t|<x|<y|<z| \mathcal{O}|z\rangle|y\rangle|x\rangle|t\rangle .
$$

Eq. (5) can be written as

$$
W=\frac{i}{2} \int_{0}^{\infty} \frac{d s}{s} \operatorname{Tr}\left[e^{i s\left[\left(\delta_{j k} \hat{p}-g T_{j k}^{a} A^{a}\right)^{2}+\frac{g}{2} \sigma^{\mu \nu} T_{j k}^{a} F_{\mu \nu}^{a}-\delta_{j k} m^{2}+i \epsilon\right]}-e^{i s\left[\delta_{j k}\left(\hat{p}^{2}-m^{2}\right)+i \epsilon\right]}\right] .
$$

\section{A. Suitable Gauge Choice for Evaluation of this Path Integral}

A physical quantity constructed from the arbitrary space-dependent static color potential

$$
A_{\mu}^{a}(x)=-\delta_{\mu 0} \int d x E^{a}(x)
$$


may be expressed in terms of two space-dependent casimir/gauge invariants in $\mathrm{SU}(3)$

$$
C_{1}(x)=\left[E^{a}(x) E^{a}(x)\right], \quad \text { and }, \quad C_{2}(x)=\left[d_{a b c} E^{a}(x) E^{b}(x) E^{c}(x)\right]^{2} .
$$

However, it is not possible to perform the above path integration by directly using eq. (8) in eq. (7). For this reason we use the gauge invariance argument. Since $A_{\mu}^{a}(x)$ is not gauge invariant we can work in a different gauge to obtain the same $E^{a}(x)$. For example, if we choose

$$
A_{\mu}^{a}(t, x)=-\delta_{\mu 1} E^{a}(x) t
$$

then we reproduce the same chromo-electric field $E^{a}(x)$ as in eq. (8). Note that both the equations (8) and (10) are related by a gauge transformation and reproduce the same $E^{a}(x)$ but eq. (10) is preferred to do path integration because the 't' variable will allow us to cast this as a harmonic oscillator problem. This is not possible by eq. (8) . Hence we can use eq. (10) instead of eq. (8) to perform path integration in the presence of arbitrary space-dependent static color potential.

Using eq. (10) in (7) we find

$$
\begin{aligned}
& W=\frac{i}{2} \operatorname{tr}_{\text {Dirac }} \operatorname{tr}_{\text {color }} \int_{0}^{\infty} \frac{d s}{s} \int d t \int d x \int d y \int d z<t|<x|<y|<z| \\
& {\left[e^{i s\left[-\left(\delta_{j k} \hat{p}_{1}+g T_{j k}^{a} E(x) t\right)^{2}+\delta_{j k}\left(\hat{p}_{0}^{2}-\hat{p}_{y}^{2}-\hat{p}_{z}^{2}\right)+i g \gamma^{0} \gamma^{1} T_{j k}^{a} E^{a}(x)-\delta_{j k} m^{2}+i \epsilon\right]}-e^{i s\left(\delta_{j k}\left(\hat{p}^{2}-m^{2}\right)+i \epsilon\right)}\right]} \\
& |z>| y>|x>| t>.
\end{aligned}
$$

Inserting complete set of $\mid p>$ states $\left(\int d p|p><p|=1\right)$ as appropriate we obtain (we use the normalization $<q \mid p>=\frac{1}{\sqrt{2 \pi}} e^{i q p}$ )

$$
\begin{aligned}
& W=\frac{i}{2(2 \pi)^{2}} \operatorname{Tr}_{\text {Dirac }} \operatorname{Tr}_{\text {color }}\left[\int_{0}^{\infty} \frac{d s}{s} \int_{-\infty}^{+\infty} d y \int_{-\infty}^{+\infty} d z \int d p_{y} \int d p_{z} e^{-i s\left(p_{y}^{2}+p_{z}^{2}+m^{2}-i \epsilon\right)} \int d x \int d t\right. \\
& \left.\left[<x|<t| e^{i s\left[-\left(\frac{\delta_{j k}}{i} \frac{d}{d x}+g T_{j k}^{a} E^{a}(x) t\right)^{2}+\delta_{j k} \hat{p}_{0}^{2}+i g \gamma^{0} \gamma^{1} T_{j k}^{a} E^{a}(x)\right]}|t>| x>-\frac{\delta_{j k}}{s}\right]\right] .
\end{aligned}
$$

To perform the Dirac trace we use the eigenvalues of the Dirac matrix

$$
\left(\gamma^{0} \gamma^{1}\right)_{\text {eigenvalues }}=\left(\lambda_{1}, \lambda_{2}, \lambda_{3}, \lambda_{4}\right)=(1,1,-1,-1)
$$

and find

$$
\begin{aligned}
& W=\frac{i}{2(2 \pi)^{2}} \sum_{l=1}^{4} \int_{0}^{\infty} \frac{d s}{s} \int_{-\infty}^{+\infty} d y \int_{-\infty}^{+\infty} d z \int d p_{y} \int d p_{z} e^{-i s\left(p_{y}^{2}+p_{z}^{2}+m^{2}-i \epsilon\right)} \int d x \int d t \\
& {\left[F_{l}-\frac{3}{s}\right]}
\end{aligned}
$$


where

$$
F_{l}=\operatorname{tr}_{\text {color }} \int_{-\infty}^{+\infty} d x<x\left|\int_{-\infty}^{+\infty} d t<t\right| e^{i s\left[-\left(\frac{\delta_{j k}}{i} \frac{d}{d x}+g T_{j k}^{a} E^{a}(x) t\right)^{2}+\delta_{j k} \hat{p}_{0}^{2}+i g \lambda_{l} T_{j k}^{a} E^{a}(x)\right]}|t>| x>.
$$

In the matrix notation we write the above equation as

$$
F_{l}=\operatorname{tr}_{\text {color }}\left[\int_{-\infty}^{+\infty} d x<x\left|\int_{-\infty}^{+\infty} d t<t\right| e^{i s\left[-\left(\frac{1}{i} \frac{d}{d x}+g M(x) t\right)^{2}+\hat{p}_{0}^{2}+i g \lambda_{l} M(x)\right]}|t>| x>\right]_{j k}
$$

where

$$
M_{j k}(x)=T_{j k}^{a} E^{a}(x) .
$$

\section{B. Trouble with the Color Trace Evaluation in this Path Integral}

Unlike the constant chromo-electric field case [5] it is not straight forward to take the color trace by diagonalizing the color matrix $T_{j k}^{a} E^{a}(x)$ by an orthogonal matrix $U_{j k}(x)$. This is because the orthogonal matrix $U_{j k}(x)$ is $x$ dependent and does not commute with $\frac{d}{d x}$. This was not a problem for constant chromo-electric field $E^{a}$ case [5] because the orthogonal matrix $U_{j k}$ was space-time independent. This trouble was also not there in Schwinger mechanism study in QED [6] because there are no colors in QED. This implies that as long as the derivative operator $\frac{d}{d x}$ is present in the expression for $F_{l}$ we can not take the color trace by diagonalizing the space-dependent color matrix $M_{j k}(x)=T_{j k}^{a} E^{a}(x)$ by an orthogonal matrix $U_{j k}(x)$. We will come back to this color trace issue later.

We note that since $M(x)$ and $t$ in eq. (16) commutes with each other one can not change the integration variable from $t$ to $t^{\prime}$ via: $t=t^{\prime}-\frac{1}{i g M(x)} \frac{d}{d x}$ in color space. This is because $M(x)$ and $\frac{d}{d x}$ do not commute with each other. To deal with the $x$-dependent color matrix $M_{j k}(x)$ and $\delta_{j k} \frac{d}{d x}$ we use the similarity transformation of $t$ in the color space

$$
\left[t \pm \frac{1}{i g M(x)} \frac{d}{d x}\right]_{j k}=\left[e^{ \pm \frac{1}{i g M(x)} \frac{d}{d x} \frac{d}{d t}} t e^{\mp \frac{1}{i g M(x)} \frac{d}{d x} \frac{d}{d t}}\right]_{j k}
$$

which gives

$$
\left[g M(x) t+\frac{1}{i} \frac{d}{d x}\right]_{j k}=\left[e^{\frac{1}{i g M(x)} \frac{d}{d x} \frac{d}{d t}}\left[e^{-\frac{1}{i g M(x)} \frac{d}{d x} \frac{d}{d t}} g M(x) e^{\frac{1}{i g M(x)} \frac{d}{d x} \frac{d}{d t}} t\right] e^{-\frac{1}{i g M(x)} \frac{d}{d x} \frac{d}{d t}}\right]_{j k} .
$$

Using this in eq. (16) we find

$$
\begin{aligned}
& F_{l}=\operatorname{tr}_{\text {color }}\left[\int_{-\infty}^{+\infty} d x<x\left|\int_{-\infty}^{+\infty} d t<t\right| e^{\frac{1}{i g M(x)} \frac{d}{d x} \frac{d}{d t}}\right. \\
& e^{i s\left[-\left[e^{-\frac{1}{i g M(x)} \frac{d}{d x} \frac{d}{d t}} g M(x) e^{\frac{1}{i g M(x)} \frac{d}{d x} \frac{d}{d t}} t\right]^{2}+\hat{p}_{0}^{2}+i g \lambda_{l} e^{-\frac{1}{i g M(x)} \frac{d}{d x} d t} M(x) e^{\frac{1}{i g M(x)} \frac{d}{d x} \frac{d}{d t}}\right]} \\
& \left.e^{-\frac{1}{i g M(x)} \frac{d}{d x} \frac{d}{d t}}|t>| x>\right]_{j k} .
\end{aligned}
$$


It can be seen that eq. (16) contains the product $t M(x)$ which satisfies

$$
t M(x)=M(x) t
$$

because $M(x)$ is independent of $t$. However, when we change the variable

$$
t=t^{\prime}-\frac{1}{i g M(x)} \frac{d}{d x}
$$

we find

$$
t M(x) \neq M(x) t
$$

Hence one can not change the variable $t$ by eq. (22) in (16). However, in eq. (20) there is no product $t M(x)$ present. Hence we can change the integration variable $t$ to $t^{\prime}$ via: $t=t^{\prime}-\frac{1}{i g M(x)} \frac{d}{d x}$ in the color space in eq. (20). Since the $t$ integration limit is from $-\infty$ to $+\infty$, the $t^{\prime}$ integration limit also remains from $-\infty$ to $+\infty$. Hence we find from eq. (20)

$$
\begin{aligned}
& F_{l}=\operatorname{tr}_{\text {color }}\left[\int_{-\infty}^{+\infty} d x<x\left|\int_{-\infty}^{+\infty} d t<t-\frac{1}{i g M(x)} \frac{d}{d x}\right| e^{\frac{1}{i g M(x)} \frac{d}{d x} \frac{d}{d t}}\right. \\
& e^{i s\left[-\left[e^{-\frac{1}{i g M(x)} \frac{d}{d x} \frac{d}{d t}} g M(x) e^{\frac{1}{i g M(x)} \frac{d}{d x} \frac{d}{d t}}\left(t-\frac{1}{i g M(x)} \frac{d}{d x}\right)\right]^{2}+\hat{p}_{0}^{2}+i g \lambda_{l} e^{-\frac{1}{i g M(x)} \frac{d}{d x} \frac{d}{d t}} M(x) e^{\frac{1}{i g M(x)} \frac{d}{d x} \frac{d}{d t}}\right]} \\
& \left.e^{-\frac{1}{i g M(x)} \frac{d}{d x} \frac{d}{d t}}\left|t-\frac{1}{i g M(x)} \frac{d}{d x}>\right| x>\right]_{j k} .
\end{aligned}
$$

Using the similarity transformation of $\left[t-\frac{1}{i g M(x)} \frac{d}{d x}\right]_{j k}$ from eq. (18) we find from the above equation

$$
\begin{aligned}
& F_{l}=\operatorname{tr}_{\text {color }}\left[\int_{-\infty}^{+\infty} d x<x\left|\int_{-\infty}^{+\infty} d t<t-\frac{1}{i g M(x)} \frac{d}{d x}\right| e^{\frac{1}{i g M(x)} \frac{d}{d x} \frac{d}{d t}}\right. \\
& e^{i s\left[-\left[e^{-\frac{1}{i g M(x)} \frac{d}{d x} \frac{d}{d t}} g M(x) e^{\frac{1}{i g M(x)} \frac{d}{d x} \frac{d}{d t}} e^{-\frac{1}{i g M(x)} \frac{d}{d x} \frac{d}{d t}} t e^{\frac{1}{i g M(x)} \frac{d}{d x} \frac{d}{d t}}\right]^{2}+\hat{p}_{0}^{2}+i g \lambda_{l} e^{-\frac{1}{i g M(x)} \frac{d}{d x} \frac{d}{d t}} M(x) e^{\frac{1}{i g M(x)} \frac{d}{d x} \frac{d}{d t}}\right]} \\
& \left.e^{-\frac{1}{i g M(x)} \frac{d}{d x} \frac{d}{d t}}\left|t-\frac{1}{i g M(x)} \frac{d}{d x}>\right| x>\right]_{j k}
\end{aligned}
$$

which gives

$$
\begin{aligned}
& F_{l}=\operatorname{tr}_{\text {color }}\left[\int_{-\infty}^{+\infty} d x<x\left|\int_{-\infty}^{+\infty} d t<t-\frac{1}{i g M(x)} \frac{d}{d x}\right| e^{i s\left[-g^{2} M^{2}(x) t^{2}+\hat{p}_{0}^{2}+i g \lambda_{l} M(x)\right]} \mid t-\frac{1}{i g M(x)} \frac{d}{d x}>\right. \\
& \mid x>]_{j k} .
\end{aligned}
$$

It has to be remembered that eq. (26) is not valid if the $t$ integration limit was finite [4]. Hence we must perform the $t$ integration from $-\infty$ to $+\infty$ in $F_{l}$ which we will do later in the derivation. 
Inserting complete set of $\mid p_{0}>$ states we find

$$
\begin{aligned}
& F_{l}=\operatorname{tr}_{\text {color }}\left[\int_{-\infty}^{+\infty} d x<x\left|\int_{-\infty}^{+\infty} d t \int d p_{0}^{\prime} \int d p_{0}^{\prime \prime}<t-\frac{1}{i g M(x)} \frac{d}{d x}\right| p_{0}^{\prime}>\right. \\
& \left.<p_{0}^{\prime}\left|e^{i s\left[-g^{2} M^{2}(x) t^{2}+\hat{p}_{0}^{2}+i g \lambda_{l} M(x)\right]}\right| p_{0}^{\prime \prime}><p_{0}^{\prime \prime}\left|t-\frac{1}{i g M(x)} \frac{d}{d x}>\right| x>\right]_{j k} \\
& =\frac{1}{2 \pi} \operatorname{tr}_{\text {color }}\left[\int_{-\infty}^{+\infty} d x<x\left|\int_{-\infty}^{+\infty} d t \int d p_{0}^{\prime} \int d p_{0}^{\prime \prime} e^{i t p_{0}^{\prime}} e^{-\frac{p_{0}^{\prime}}{g M(x)} \frac{d}{d x}}<p_{0}^{\prime}\right| e^{i s\left[-g^{2} M^{2}(x) t^{2}+\hat{p}_{0}^{2}+i g \lambda_{l} M(x)\right]} \mid p_{0}^{\prime \prime}>\right. \\
& \left.e^{-i t p_{0}^{\prime \prime}} e^{\frac{p_{0}^{\prime \prime}}{g M(x)} \frac{d}{d x}} \mid x>\right]_{j k} .
\end{aligned}
$$

The above equation contains $\frac{d}{d x}$ and hence we can not perform the color trace by diagonalizing the color matrix $M_{j k}(x)$ by an orthogonal matrix $U_{j k}(x)$.

Inserting complete sets of states as appropriate we find

$$
\begin{aligned}
& F_{l}=\frac{1}{2 \pi} \operatorname{tr}_{\text {color }}\left[\int_{-\infty}^{+\infty} d x \int d x^{\prime} \int d x^{\prime \prime} \int d p_{x} \int d p_{x}^{\prime} \int d p_{x}^{\prime \prime} \int d p_{x}^{\prime \prime \prime}<x \mid p_{x}>\int_{-\infty}^{+\infty} d t \int d t^{\prime} \int d t^{\prime \prime}\right. \\
& \int d p_{0}^{\prime} \int d p_{0}^{\prime \prime} e^{i t p_{0}^{\prime}}<p_{x}\left|e^{-\frac{1}{g M(x)} \frac{d}{d x} p_{0}^{\prime}}\right| p_{x}^{\prime}><p_{0}^{\prime}\left|t^{\prime}><p_{x}^{\prime}\right| x^{\prime}><x^{\prime}\left|<t^{\prime}\right| e^{i s\left[-g^{2} M^{2}(x) t^{2}+\hat{p}_{0}^{2}+i g \lambda_{l} M(x)\right]} \\
& \left.\left|t^{\prime \prime}>\right| x^{\prime \prime}><x^{\prime \prime}\left|p_{x}^{\prime \prime}><t^{\prime \prime}\right| p_{0}^{\prime \prime}><p_{x}^{\prime \prime}\left|e^{\frac{1}{g(x)} \frac{d}{d x} p_{0}^{\prime \prime}}\right| p_{x}^{\prime \prime \prime}>e^{-i t p_{0}^{\prime \prime}}<p_{x}^{\prime \prime \prime} \mid x>\right]_{j k} .
\end{aligned}
$$

Since the matrix $\left[<p_{x}^{\prime \prime}\left|e^{\frac{1}{g M(x)} \frac{d}{d x} p_{0}^{\prime \prime}}\right| p_{x}^{\prime \prime \prime}>\right]_{j k}$ is inside the trace and is independent of $\frac{d}{d x}$ (it depends on c-numbers $p_{x}^{\prime \prime}$ and $\left.p_{x}^{\prime \prime \prime}\right)$, we can move it to the left. We find

$$
\begin{aligned}
& F_{l}=\frac{1}{(2 \pi)^{4}} \operatorname{tr}_{\text {color }}\left[\int_{-\infty}^{+\infty} d x \int d x^{\prime} \int d p_{x} \int d p_{x}^{\prime} \int d p_{x}^{\prime \prime} \int d p_{x}^{\prime \prime \prime} e^{i x p_{x}} \int_{-\infty}^{+\infty} d t \int d t^{\prime} \int d t^{\prime \prime}\right. \\
& \int d p_{0}^{\prime} \int d p_{0}^{\prime \prime} e^{i t p_{0}^{\prime}}<p_{x}^{\prime \prime}\left|e^{\frac{1}{g M(x)} \frac{d}{d x} p_{0}^{\prime \prime}}\right| p_{x}^{\prime \prime \prime}><p_{x}\left|e^{-\frac{1}{g M(x)} \frac{d}{d x} p_{0}^{\prime}}\right| p_{x}^{\prime}>e^{-i p_{0}^{\prime} t^{\prime}} e^{-i x^{\prime} p_{x}^{\prime}} \\
& \left.<t^{\prime}\left|e^{i s\left[-g^{2} M^{2}\left(x^{\prime}\right) t^{2}+\hat{p}_{0}^{2}+i g \lambda_{l} M\left(x^{\prime}\right)\right]}\right| t^{\prime \prime}>e^{i x^{\prime} p_{x}^{\prime \prime}} e^{i p_{0}^{\prime \prime} t^{\prime \prime}} e^{-i t p_{0}^{\prime \prime}} e^{-i x p_{x}^{\prime \prime \prime}}\right]_{j k} .
\end{aligned}
$$

The matrix $\left[<p_{x}^{\prime \prime}\left|e^{\frac{1}{g M(x)} \frac{d}{d x} p_{0}^{\prime \prime}}\right| p_{x}^{\prime \prime \prime}>\right]_{j k}$ is also independent of $x$. This can be shown as follows

$$
\begin{aligned}
& <p_{x}^{\prime \prime}\left|f(x) \frac{d}{d x}\right| p_{x}^{\prime \prime \prime}>=\int d x^{\prime} \int d x^{\prime \prime} \int d p_{x}^{\prime \prime \prime \prime}<p_{x}^{\prime \prime}\left|x^{\prime}><x^{\prime}\right| f(x)\left|x^{\prime \prime}><x^{\prime \prime}\right| p_{x}^{\prime \prime \prime \prime}><p_{x}^{\prime \prime \prime \prime}\left|\frac{d}{d x}\right| p_{x}^{\prime \prime \prime}> \\
& =\int d x^{\prime} e^{-i x^{\prime}\left(p_{x}^{\prime \prime}-p_{x}^{\prime \prime \prime}\right)} f\left(x^{\prime}\right) i p_{x}^{\prime \prime \prime}
\end{aligned}
$$

which is independent of $x$ and $\frac{d}{d x}$. Hence all the expressions in eq. (29) are independent of $x$ except $e^{i x\left(p_{x}-p_{x}^{\prime \prime \prime}\right)}$. This implies that we can easily perform the $x$ integration in eq. (29) (by using $\left.\int_{-\infty}^{+\infty} d x e^{i x\left(p_{x}-p_{x}^{\prime \prime \prime}\right)}=2 \pi \delta_{(} p_{x}-p_{x}^{\prime \prime \prime}\right)$ ) to obtain

$$
\begin{aligned}
& F_{l}=\frac{1}{(2 \pi)^{3}} \operatorname{tr}_{\text {color }}\left[\int d x^{\prime} \int d p_{x} \int d p_{x}^{\prime} \int d p_{x}^{\prime \prime} \int_{-\infty}^{+\infty} d t \int d t^{\prime} \int d t^{\prime \prime} \int d p_{0}^{\prime} \int d p_{0}^{\prime \prime}\right. \\
& e^{i t p_{0}^{\prime}}<p_{x}^{\prime \prime}\left|e^{\frac{1}{g M(x)} \frac{d}{d x} p_{0}^{\prime \prime}}\right| p_{x}><p_{x}\left|e^{-\frac{1}{g M(x)} \frac{d}{d x} p_{0}^{\prime}}\right| p_{x}^{\prime}>e^{-i p_{0}^{\prime} t^{\prime}} \\
& \left.e^{-i x^{\prime} p_{x}^{\prime}}<t^{\prime}\left|e^{i s\left[-g^{2} M^{2}\left(x^{\prime}\right) t^{2}+\hat{p}_{0}^{2}+i g \lambda_{l} M\left(x^{\prime}\right)\right]}\right| t^{\prime \prime}>e^{i x^{\prime} p_{x}^{\prime \prime}} e^{i p_{0}^{\prime \prime} t^{\prime \prime}} e^{-i t p_{0}^{\prime \prime}}\right]_{j k} .
\end{aligned}
$$


As advocated earlier in eq. (26) we must integrate over $t$ from $-\infty$ to $+\infty$ in $F_{l}$. Since $<t^{\prime}\left|e^{i s\left[-g^{2} M^{2}\left(x^{\prime}\right) t^{2}+\hat{p}_{0}^{2}+i g \lambda_{l} M\left(x^{\prime}\right)\right]}\right| t^{\prime \prime}>$ is independent of $t$ variable (it depends on $t^{\prime}$ and $t^{\prime \prime}$ variables) all the expressions in eq. (31) are independent of $t$ except $e^{i t\left(p_{0}^{\prime}-p_{0}^{\prime \prime}\right)}$. Hence we can easily integrate over $t$ (by using $\left.\int_{-\infty}^{+\infty} d t e^{i t\left(p_{0}^{\prime}-p_{0}^{\prime \prime}\right)}=2 \pi \delta\left(p_{0}^{\prime}-p_{0}^{\prime \prime}\right)\right)$ to obtain

$$
\begin{aligned}
& F_{l}=\frac{1}{(2 \pi)^{2}} \operatorname{tr}_{\text {color }}\left[\int d x^{\prime} \int d p_{x} \int d p_{x}^{\prime} \int d p_{x}^{\prime \prime} \int d t^{\prime} \int d t^{\prime \prime} \int d p_{0}^{\prime}\right. \\
& <p_{x}^{\prime \prime}\left|e^{\frac{1}{g M(x)} \frac{d}{d x} p_{0}^{\prime}}\right| p_{x}><p_{x}\left|e^{-\frac{1}{g M(x)} \frac{d}{d x} p_{0}^{\prime}}\right| p_{x}^{\prime}>e^{-i p_{0}^{\prime} t^{\prime}} \\
& \left.e^{-i x^{\prime} p_{x}^{\prime}}<t^{\prime}\left|e^{i s\left[-g^{2} M^{2}\left(x^{\prime}\right) t^{2}+\hat{p}_{0}^{2}+i g \lambda_{l} M\left(x^{\prime}\right)\right]}\right| t^{\prime \prime}>e^{i x^{\prime} p_{x}^{\prime \prime}} e^{i p_{0}^{\prime} t^{\prime \prime}}\right]_{j k} .
\end{aligned}
$$

Since $\int d p_{x}\left|p_{x}><p_{x}\right|=1$ we find from the above equation

$$
\begin{aligned}
& F_{l}=\frac{1}{(2 \pi)^{2}} \operatorname{tr}_{\text {color }}\left[\int d x^{\prime} \int d p_{x}^{\prime} \int d t^{\prime} \int d t^{\prime \prime} \int d p_{0}^{\prime} e^{-i t^{\prime} p_{0}^{\prime}}\right. \\
& \left.<t^{\prime}\left|e^{i s\left[-g^{2} M^{2}\left(x^{\prime}\right) t^{2}+\hat{p}_{0}^{2}+i g \lambda_{l} M\left(x^{\prime}\right)\right]}\right| t^{\prime \prime}>e^{i t^{\prime \prime} p_{0}^{\prime}}\right]_{j k} .
\end{aligned}
$$

\section{Evaluation of the Color Trace}

As the above equation does not contain any derivative $\frac{d}{d x}$ operator we can perform the color trace by diagonalizing the space-dependent color matrix $M_{j k}(x)$ by a space-dependent orthogonal matrix $U_{j k}(x)$. In the fundamental representation the matrix $M_{j k}(x)$ has three eigenvalues:

$$
\left[M_{j k}(x)\right]_{\text {eigenvalues }}=\left[T_{j k}^{a} E^{a}(x)\right]_{\text {eigenvalues }}=\left(\Lambda_{1}(x), \Lambda_{2}(x), \Lambda_{3}(x)\right) .
$$

Evaluating the traces of $M_{j k}(x), M_{j k}^{2}(x)$ and $M_{j k}^{3}(x)$ we find

$$
\begin{aligned}
\Lambda_{1}(x)+\Lambda_{2}(x)+\Lambda_{3}(x) & =0 \\
\Lambda_{1}^{2}(x)+\Lambda_{2}^{2}(x)+\Lambda_{3}^{2}(x) & =\frac{E^{a}(x) E^{a}(x)}{2}, \\
\Lambda_{1}^{3}(x)+\Lambda_{2}^{3}(x)+\Lambda_{3}^{3}(x) & =\frac{1}{4}\left[d_{a b c} E^{a}(x) E^{b}(x) E^{c}(x)\right]
\end{aligned}
$$

the solution of which is given by eq. (2).

Using the eigenvalues of $M_{j k}(x)$ from eq. (2) we perform the color trace in eq. (33) and find

$$
\begin{aligned}
& F_{l}=\frac{1}{(2 \pi)^{2}} \sum_{j=1}^{3}\left[\int d x \int d p_{x} \int d t^{\prime} \int d t^{\prime \prime} \int d p_{0}^{\prime} e^{-i t^{\prime} p_{0}^{\prime}}\right. \\
& \left.<t^{\prime}\left|e^{i s\left[-g^{2} \Lambda_{j}^{2}(x) t^{2}+\hat{p}_{0}^{2}+i g \lambda_{l} \Lambda_{j}(x)\right]}\right| t^{\prime \prime}>e^{i t^{\prime \prime} p_{0}^{\prime}}\right] .
\end{aligned}
$$


Since the eigenvalues $\Lambda_{j}$ 's are c-numbers, the above equation involves one harmonic oscillator

$$
\left[\frac{1}{2} \omega^{2}(x) t^{2}+\frac{1}{2} \hat{p}_{0}^{2}\right]\left|n>=\left(n+\frac{1}{2}\right) \omega(x)\right| n>
$$

with space-dependent frequency $\omega(x)$. The harmonic oscillator wave function

$$
<t \mid n>=\psi_{n}(t)=\left(\frac{\omega(x)}{\pi}\right)^{1 / 4} \frac{1}{\left(2^{n} n !\right)^{1 / 2}} H_{n}(t \sqrt{\omega(x)}) e^{-\frac{\omega(x)}{2} t^{2}}
$$

with space dependent frequency $\omega(x)$ is normalized,

$$
\int d t|<t| n>\left.\right|^{2}=1
$$

where $H_{n}$ is the Hermite polynomial. Inserting complete set of harmonic oscillator states (by using $\sum_{n}|n><n|=1$ ) in eq. (36) we find

$$
\begin{aligned}
& F_{l}=\frac{1}{(2 \pi)^{2}} \sum_{j=1}^{3} \sum_{n} \int d x \int d p_{x} \int d t^{\prime} \int d t^{\prime \prime} \int d p_{0}^{\prime} e^{-i t^{\prime} p_{0}^{\prime}}<t^{\prime} \mid n>e^{-s\left[g \Lambda_{j}(x)(2 n+1)+g \lambda_{l} \Lambda_{j}(x)\right]} \\
& <n\left|t^{\prime \prime}>e^{i t^{\prime \prime} p_{0}^{\prime}}=\frac{1}{2 \pi} \sum_{j=1}^{3} \int d x \int d p_{x} \int d t\right|<t|n>|^{2} e^{-s g \lambda_{l} \Lambda_{j}(x)} \frac{1}{2 \sinh \left(s g \Lambda_{j}(x)\right)} \\
& =\frac{1}{4 \pi} \operatorname{tr}_{\text {color }}\left[\int d x \int d p_{x} \frac{e^{-s g \lambda_{l} M(x)}}{\sinh (s g M(x))}\right]_{j k}
\end{aligned}
$$

where we have used eq. (39). The Lorentz force equation: $\delta_{j k} d p^{\mu}=g T_{j k}^{a} F^{a \mu \nu}(x) d x_{\nu}$, of the quark in color space becomes (when $E^{a}(x)$ is along the $x$-axis, eq. (10))

$$
\delta_{j k} d p_{x}=g T_{j k}^{a} E^{a}(x) d t=g M_{j k}(x) d t .
$$

Implementing this in eq. (40) we find

$$
F_{l}=\frac{1}{4 \pi} \sum_{j=1}^{3} \int d x \int d t \frac{g \Lambda_{j}(x) e^{-s g \lambda_{l} \Lambda_{j}(x)}}{\sinh \left(s g \Lambda_{j}(x)\right)} .
$$

\section{The Generating Functional}

Using the above expression of $F_{l}$ in eq. (14) we obtain

$$
W=\frac{i}{32 \pi^{3}} \sum_{l=1}^{4} \sum_{j=1}^{3} \int_{0}^{\infty} \frac{d s}{s} \int d t \int d^{3} x \int d^{2} p_{T} e^{-i s\left(p_{T}^{2}+m^{2}-i \epsilon\right)}\left[\frac{g \Lambda_{j}(x) e^{-s g \lambda_{l} \Lambda_{j}(x)}}{\sinh \left(s g \Lambda_{j}(x)\right)}-\frac{1}{s}\right]
$$


where $p_{T}^{2}=p_{y}^{2}+p_{z}^{2}$. Summing over $l$, by using the eigenvalues $\left(\lambda_{l}\right)$ of the Dirac matrix $\gamma^{0} \gamma^{1}$ from eq. (13) and integrating over $p_{T}$ we find

$$
W=\frac{1}{8 \pi^{2}} \sum_{j=1}^{3} \int d t \int d^{3} x \int_{0}^{\infty} \frac{d s}{s^{2}} e^{-i s\left(m^{2}-i \epsilon\right)}\left[g \Lambda_{j}(x) \operatorname{coth}\left(g s \Lambda_{j}(x)\right)-\frac{1}{s}\right] .
$$

This equation is divergent as $s \rightarrow 0$. This ultraviolet divergence can be removed by charge renormalization [7] by subtracting also the term linear in $s$ in the expansion of $\frac{\cosh \left(\operatorname{sg} \Lambda_{j}(x)\right)}{\sinh \left(\operatorname{sg} \Lambda_{j}(x)\right)}$. Hence after renormalization we obtain

$$
W=\frac{1}{8 \pi^{2}} \sum_{j=1}^{3} \int d t \int d^{3} x \int_{0}^{\infty} \frac{d s}{s^{2}} e^{-i s\left(m^{2}-i \epsilon\right)}\left[g \Lambda_{j}(x) \operatorname{coth}\left(g s \Lambda_{j}(x)\right)-\frac{1}{3} s g^{2} \Lambda_{j}^{2}(x)-\frac{1}{s}\right] .
$$

Using eq. (45) in (44) we find the following expression for the generating functional of quark (antiquark) in the presence of arbitrary space-dependent static color potential $A_{0}^{a}(x)=-\int d x E^{a}(x)$ with arbitrary color index $a=1,2, \ldots 8$ in $\mathrm{SU}(3)$,

$$
\begin{aligned}
& \frac{Z[A]}{Z[0]}=\frac{\int[d \bar{\psi}][d \psi] e^{i \int d^{4} x \bar{\psi}^{j}(x)\left(\delta_{j k} \not{p}-g T_{j k}^{a} \not^{a}-\delta_{j k} m\right) \psi^{k}(x)}}{\int[d \bar{\psi}][d \psi] e^{i \int d^{4} x \bar{\psi}^{j}(x)(\not{p}-m) \psi^{k}(x)}}= \\
& \exp \left\{\frac{i}{8 \pi^{2}} \sum_{j=1}^{3} \int d t \int d^{3} x \int_{0}^{\infty} \frac{d s}{s^{2}} e^{-i s\left(m^{2}-i \epsilon\right)}\left[g \Lambda_{j}(x) \operatorname{coth}\left(g s \Lambda_{j}(x)\right)-\frac{1}{3} s g^{2} \Lambda_{j}^{2}(x)-\frac{1}{s}\right]\right\}
\end{aligned}
$$

which reproduces eq. (11). The gauge invariant expressions for $\Lambda_{j}(x)$ 's are given in eq. (2)).

\section{CONCLUSION}

To conclude we have performed the path integral for a quark (antiquark) in the presence of arbitrary space-dependent static color potential $A_{0}^{a}(x)\left(=-\int d x E^{a}(x)\right)$ with arbitrary color index $a=1,2, \ldots 8$. We have shown that such a path integration is possible even if one can not solve the Dirac equation in the presence of arbitrary space-dependent potential. In particular we have obtained an exact expression of the non-perturbative generating functional of quark (antiquark) in the presence of arbitrary space-dependent color potential $A_{0}^{a}(x)\left(=-\int d x E^{a}(x)\right)$ with arbitrary color index $a=1,2, \ldots 8$ in $\mathrm{SU}(3)$. This result crucially depends on the validity of the shift conjecture which has not yet been established.

It may be possible to further explore this path integral technique to study nonperturbative bound state formation, for example, by studying various non-perturbative correlation functions. 


\section{Acknowledgments}

I thank Robert Shrock, Jack Smith and George Sterman for useful discussions. I also thank Jack Smith and George Sterman for careful reading of the manuscript. This work was supported in part by the National Science Foundation, grants PHY-0354776 and PHY0345822 .

[1] K. G. Wilson, Phys. Rev. D10 (1974) 2445.

[2] H. J. Rothe, "Lattice Gauge Theories", world scientific lecture notes in physics-vol. 74, (2005); and references therein.

[3] J. D. Stack, Phys. Rev. D27 (1983) 412; Phys. Rev. D29 (1984) 1213; H.-Q. Ding, Phys. Rev. D42 (1990) 2350; M. Baker, J. S. Ball and F. Zachariasen, Phys. Rev. D56 (1997) 4400; and and references therein.

[4] F. Cooper and G. C. Nayak, hep-th/0609192.

[5] G. C. Nayak, Phys. Rev. D72 (2005) 125010; G. C. Nayak and P. van Nieuwenhuizen, Phys. Rev. D 71 (2005) 125001; F. Cooper and G. C. Nayak, Phys. Rev. D 73 (2006) 065005.

[6] F. Cooper and G. C. Nayak, hep-th/0611125; hep-th/0612292.

[7] J. Schwinger, Phys. Rev. 82 (1951) 664. 